\title{
The transcriptome of nitrofen-induced pulmonary hypoplasia in the rat model of congenital diaphragmatic hernia
}

\author{
Thomas H. Mahood', Dina R. Johar ${ }^{1,2}$, Barbara M. Iwasiow ${ }^{1,3}$, Wayne X $\mathbf{u}^{4,5}$ and Richard Keijzer ${ }^{1,3}$
}

BACKGROUND: We currently do not know how the herbicide nitrofen induces lung hypoplasia and congenital diaphragmatic hernia in rats. Our aim was to compare the differentially expressed transcriptome of nitrofen-induced hypoplastic lungs to control lungs in embryonic day 13 rat embryos before the development of embryonic diaphragmatic defects.

METHODS: Using next-generation sequencing technology, we identified the expression profile of microRNA (miRNA) and mRNA genes. Once the dataset was validated by both RT-qPCR and digital-PCR, we conducted gene ontology, miRNA target analysis, and orthologous miRNA sequence matching for the deregulated miRNAs in silico.

RESULTS: Our study identified 186 known mRNA and 100 miRNAs which were differentially expressed in nitrofeninduced hypoplastic lungs. Sixty-four rat miRNAs homologous to known human miRNAs were identified. A subset of these genes may promote lung hypoplasia in rat and/or human, and we discuss their associations. Potential miRNA pathways relevant to nitrofen-induced lung hypoplasia include PI3K, TGF- $\beta$, and cell cycle kinases.

CONCLUSION: Nitrofen-induced hypoplastic lungs have an abnormal transcriptome that may lead to impaired development.

M icroRNAs (miRNAs) are small (17-24bp), noncoding RNAs that regulate different biological functions by modulating expression and stability of target genes posttranscriptionally $(1,2)$. The role of miRNAs in lung development is currently poorly defined. Deregulation of miRNAs, and consequently, loss of fine adjustments to target genes can lead to lung diseases (3).

Babies with congenital diaphragmatic hernia $(\mathrm{CDH})$ have a hole in their diaphragm and abnormal lung development resulting in lung hypoplasia and persistent pulmonary hypertension. $\mathrm{CDH}$ constitutes about $8 \%$ of major congenital anomalies and has the highest mortality rate (4). In rodents, nitrofen induces $\mathrm{CDH}$ and lung hypoplasia similar to the phenotype observed in humans (5). Whether the nitrofen-rat model shares the same pathogenesis with human $\mathrm{CDH}$ remains to be determined. Some hypotheses suggest that nitrofen influences diaphragm and lung development in rodent embryos similar to the human condition (6-8). Thus far, the transcriptomic changes associated with nitrofen-modulated developmental signaling have not been characterized in the lung.

In this study, we used next-generation sequencing (NGS) technology to characterize transcriptome differences between control lungs and nitrofen-induced hypoplastic lungs in embryonic lung tissues prior to the development of the diaphragmatic defect. We hypothesize that transcriptome changes contribute to abnormal lung development in the nitrofen rat model of CDH. Exploring such phenotypic mechanisms in rats could help improve our understanding of abnormal lung development in human $\mathrm{CDH}$.

\section{RESULTS}

NGS Sequencing Results

A total of 5,045,279 single-end (50bp) MiSeq miRNA sequence reads were generated and $153,253,385$ pair-end $(50 \times 35)$ SOLiD sequence mRNA reads were generated from nitrofentreated and control lung samples. Over $90 \%$ of the mRNA, reads were mapped on the Rattus norvegicus reference genome (rn5) using Lifescope v2.5.1 software (ThermoFisher, Grand Island, NY) with two-mismatch settings.

\section{miRNA and mRNA Profile Induced by Nitrofen}

Once the MiSeq sequences were normalized, a large number of unaligned sequences were identified possibly due to the low abundance of embryonic RNA. Due to the nature of working with limited material derived from embryonic animals, a pooled sample collection was analyzed to strengthen our alignment profile. To compensate for the pooled effects of the data, a strict statistical filtering of the raw miRNA dataset provided a focused approach identifying differentially expressed miRNAs

\footnotetext{
The first two authors contributed equally to this work.

'Department of Physiology and Pathophysiology, University of Manitoba and Children's Hospital Research Institute of Manitoba, Winnipeg, Manitoba, Canada; ${ }^{2}$ Ain Shams University Faculty of Women for Arts, Sciences and Education, Cairo, Egypt; ${ }^{3}$ Department of Surgery, Paediatrics \& Child Health, University of Manitoba, Winnipeg, Manitoba, Canada; ${ }^{4}$ Department of Biochemistry and Medical Genetics, Faculty of Medicine, University of Manitoba, Winnipeg, Manitoba, Canada; ${ }^{5}$ Next Generation Sequencing Platform, Children's Hospital Research Institute of Manitoba and Manitoba Institute of Cell Biology, University of Manitoba, Winnipeg, Manitoba, Canada. Correspondence: Richard Keijzer (richardkeijzer@gmail.com)

Received 22 June 2015; accepted 29 October 2015; advance online publication 3 February 2016. doi:10.1038/pr.2015.277
} 
in the nitrofen-induced hypoplastic lungs. The dataset resulted in 100 significantly induced miRNAs $(P<0.01)$ that were differentially induced by a fold change greater than 1.5 (see Supplementary Table S1 online). The sequences of other small RNA species including ribosomal (rRNA), small nuclear, small nucleolar and transfer were annotated against the University of Santa Cruz genome browser (9) (see Supplementary Figure S1 online). Analysis of the mRNA transcriptome identified 186 significantly induced mRNA coding genes $(P<0.01)$ (see Supplementary Table S2 online). Nitrofen treatment induced 80 mRNA genes by >twofold change while 106 genes were downregulated by <onefold change relative to controls.

\section{Functional Enrichment Analysis of mRNA}

The differentially expressed transcript datasets were entered into QIAGEN's Ingenuity Pathway Analysis (IPA, QIAGEN Redwood City, CA) for mRNA (see Supplementary Table S2 online) and miRNA pathway analysis (see Supplementary Table S1 online), PANTHER Gene Ontology classification system (10) for functional gene expression analysis (Figure 1), and finally differentially expressed genes were mapped to the Kyoto Encyclopedia of Genes and Genomes (KEGG) (11). IPA analysis identified several signaling pathways including cell survival mechanisms, lipid metabolism, cellular movement and more tissue-specific mechanisms such as connective tissue development. Using PANTHER transcript classification, processes such as metabolism, protein binding, catalytic activity, WNT signaling pathway and protein functions such as transferase activity and nucleic acid binding were most apparent.

Using a binomial distribution and Log2 conversion of the gene expression data, genes were entered into KEGG (11) and set to a binomial distribution of expression (upregulation, red, downregulation, green). Pathways of significant interest included WNT, transforming growth factor (TGF)- $\beta$ signaling and retinol metabolism (Figure 2).

We grouped the identified miRNAs on the basis of their sequence similarity into 83 families. Sequence comparison revealed 64 human orthologs to previously described rat miRNAs (mirBase release 21 (12)). We identified 10 miRNA families with at least 10 sequence reads at the embryonic stage in human that are potentially relevant to the nitrofen-induced lung pathology in the rat. We identified 19 putative interacting miRNA-mRNA pairs highly relevant to nitrofen-induced lung development (Table 1). This linkage between miRNAs and mRNA was also statistically linked using IPA software.

\section{Nitrofen-induced Changes in miRNA Expression Affect Gene Transcription}

From the differentially expressed miRNAs and mRNA genes, we identified 78 differentially correlated pairs of miRNAs and mRNAs. The assessment of miRNA and mRNA expression levels revealed significant positive and negative correlations in 8 and $11 \%$ of the identified pairs, respectively (Table 1). The correlations suggest potential functional relationships that contribute to the pathogenesis of lung hypoplasia upon nitrofen treatment. Of the identified mRNA reads, EPHX3, PSG19,
HNF4a, CAR7 and KCNH7 displayed the highest significant differentially expression (fold change $>6$ ). Amongst the next 82 mRNAs that had a more moderate yet substantial upregulated expression during embryonic and fetal lung development, (fold change >2) were 14 genes: ATP5F1, CD99, PYGO2, MIP, CCDC126, STXBP6, ATP5I, FAM111A, RPI35A, TNKS2, TPMT, ID4, RPS3A, PSMA6. Also, 34 genes were downregulated (fold change <0.5) during embryonic and fetal lung development.

\section{PCR Validation of the Differential Transcripts and miRNAs}

Due to the variable nature of gene expression in developmental tissues along with the significant phenotypic changes induced by nitrofen treatment in embryonic rats, a survey of 32 commonly used endogenous genes was performed (see Supplementary Figure S2 online). Comparing both nitrofen and control E21 lungs, we identified both ABL and GAPDH as excellent endogenous control genes for RT-qPCR (CV: 0.0769, $M$ value: 0.2221 ). These two genes were used in tandem through a geometric normalization approach for all functional gene expression analysis (digital PCR, dPCR and RT-qPCR). Two-way ANOVA of results run in Prism (ver 6.0) using a Sidak repeated measures test identified that only PSG19 was significantly upregulated compared to control $(P=0.0010)$. Chromatin licensing and DNA replicating factor 1 (CDT1) was significantly downregulated due to nitrofen treatment (two-tailed unpaired $t$-test, $P=0.0420$ ). Using the same cDNA samples and primer sets from the dPCR, RT-qPCR was also conducted. As expected, a similar trend in gene expression was observed. However, no significant changes were shown for PSG19 when compared to control (two-way unpaired $t$-test, $P=0.0558)$. Using the calculated fold change in gene expression from both dPCR and RT-qPCR, a linear regression was constructed to determine the correlation between RNA-sequencing and gene expression validation (Figure 3 ). Using these methods, $\mathrm{dPCR}$ (slope $=0.592, R^{2}=0.34$ ) was shown to be a more accurate method to assess changes in gene expression to that of RT-qPCR (slope $=0.457, R^{2}=0.11$ ). Furthermore, large changes in gene expression were more strongly correlated between $\mathrm{APCR}$ and RNA sequencing compared to smaller changes.

\section{DISCUSSION}

The purpose of this study was to determine which mRNAs and miRNAs are differentially expressed in the embryonic rat lung after nitrofen treatment and which developmental signaling pathways are affected. Large changes in our gene expression data show a similar trend between NGS and functional analysis, however, smaller changes in gene expression are lost amongst the gene expression variability in this embryonic treatment despite identifying key endogenous control genes that are stable after embryonic nitrofen treatment.

\section{Conservation in Structure and Function of RNA-coding Genes Can Be used to Distinct Rat From Human in Terms of Their Responses to Nitrofen}

Some miRNA profiling studies during lung organogenesis demonstrated that the overall expression profile was similar for 


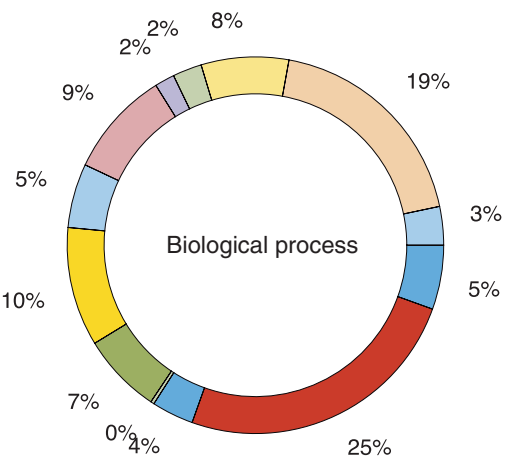
Cellular process $(\mathbf{1 8 . 8} \%)$ Localization $(7.5 \%)$

Apoptotic process $(\mathbf{2 . 5} \%)$

Reproduction (1.7\%)

Biological regulation $(\mathbf{9 . 1 \%})$

Response to stimulus $(\mathbf{5 . 5} \%)$

c

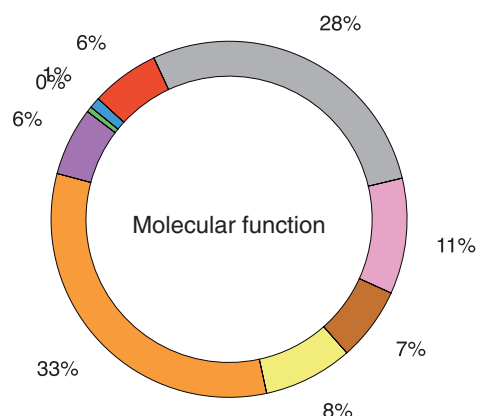

Transporter activity (6.2\%)

Translation regulator activity (1\%)

Protein-binding transcription factor activity $(0.5 \%)$

Enzyme regulator activity $(6.2 \%)$

Catalytic activity (32.5\%)

Receptor activity (8.1\%)

Stuclicacid binding transcription factor

Structural molecule activity $(\mathbf{1 0 . 5 \%}$

Binding $(\mathbf{2 8 . 2} \%)$

$7 \%$

e

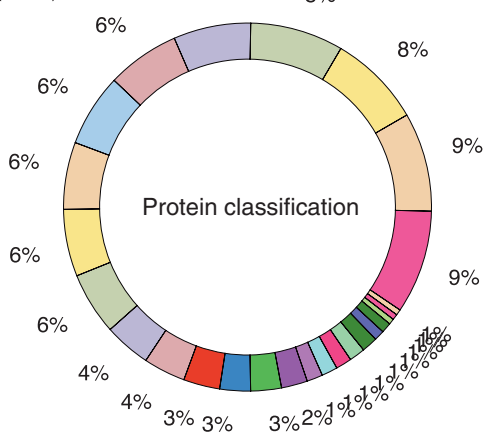

Transfer/carrier protein (2.7\%) Protease (2.7\%) Calcium-binding protein $(2.3 \%)$ Cell junction protein $(1.4$ Phosphatase (1.4\%) Defense/imm
Lyase (1.4\%) Extracellular matrix protein $(1.4 \%)$ Structural protein $(0.9 \%)$ Membrane traffic protein $(0.9 \%)$ Isomerase $(0.5 \%)$
Chaperone $(0.5 \%)$

Transmembrane receptor regulatory/adaptor protein $(0.5 \%)$ b
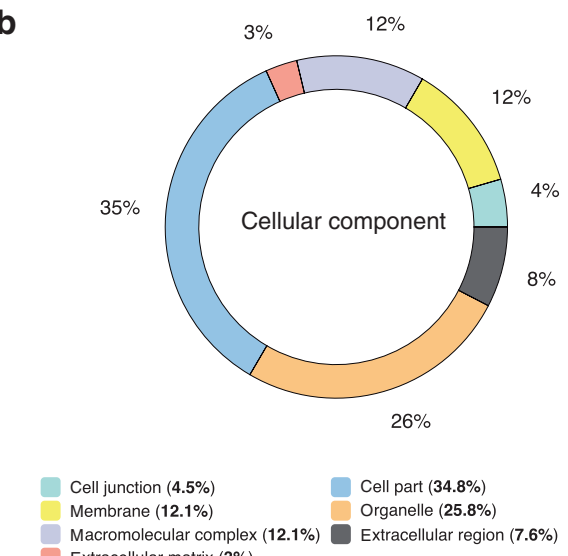

d

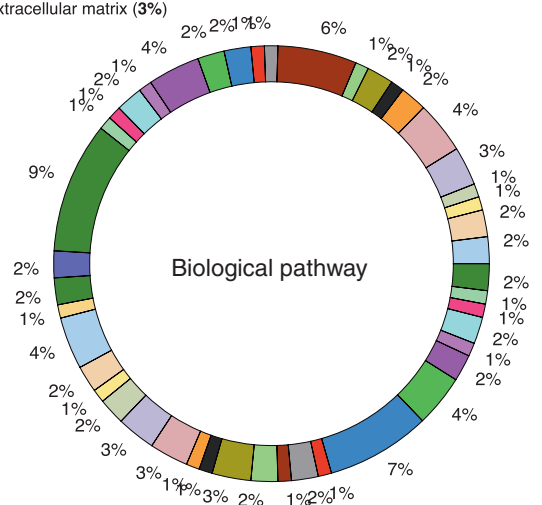

Axon guidance mediated by netrin $(2 \%)$

Axon guidance mediated by Slit/Robo $(2 \%)$

Apoptosis signaling pathway ( $1 \%$ )

JAK/STAT signaling path
Angiogenesis $(\mathbf{2 . 9 \%})$

Alzheimer disease-presenilin pathway (3.9\%)

5 HT2 type receptor-mediated signaling pathway $(2 \%)$

Coenzyme A biosynthesis ( $1 \%$ )

Integrin signalling pathway $(2 \%$

Insulin/IGF pathway-protein kinase B signaling cascade (1\%)

Inflammation mediated by chemokine and cytokine signaling pathway $(5.9 \%)$

Hypoxia response via HIF activation (1\%)

codynamics pathway $(1 \%)$

GABA-B ${ }_{r}$ eceptor ${ }_{s}$ ignaling $(2 \%)$

Huntington disease $(2 \%)$

p53 pathway $(3.9 \%)$

G-protein signaling pathway-Gq alpha and Go alpha-mediated pathway $(2 \%)$

G-protein signaling pathway-Gi alpha and Gs alpha-mediated pathway (1\%)

Wnt signaling pathway $(9.8 \%)$

VEGF signaling pathway $(2 \%)$

Thyrotropin-releasing hormone receptor signaling pathway (2\%)

T cell activation $(1 \%)$

FGF signaling pathway $(3.9 \%)$

TGF-beta signaling pathway $(2 \%)$

FAS signaling pathway $(1 \%)$

Oxytocin receptor-mediated signaling pathway $(2 \%)$

Endothelin signaling pathway $(2.9 \%)$

EGF receptor signaling pathway $(2.9 \%)$

Parkinson disease (1\%)

Cytoskeletal regulation by Rho GTPase $(2.9 \%)$

PDGF signaling pathway $(2 \%)$

Oxidative stress response $(1 \%)$

Histamine $\mathrm{H} 1$ receptor-mediated signaling pathway $(2 \%)$

Notch signaling pathway (1\%)

Cadherin signaling pathway $(\mathbf{7 . 8 \%})$

Nicotinic acetylcholine receptor signaling pathway $(3.9 \%)$

Blood coagulation $(2 \%)$

Dopamine receptor-mediated signaling pathway $(1 \%)$

B cell activation $(2 \%)$

Muscarinic acetylcholine receptor 1 and 3 signaling pathway (1\%)

Cortocotropin-releasing factor receptor signaling pathway (1\%)

Figure 1. Transcript gene functional analysis was conducted using the PANTHER gene ontology database. Gene ontology has been categorized into (a) biological processes, (b) cellular components, (c) molecular function, (d) biological pathways, and (e) protein classification.

mouse, rat, and human lung tissue, suggesting evolutionary conservation (13). MiRNA expression profiling by NGS technology has the advantage of both sensitivity, distinguishing between miRNAs that differ in sequences by as little as a single nucleotide and high throughput. Using NGS sequencing technology, we obtained reads from both the $5^{\prime}$ and $3^{\prime}$ arms of the pre-miRNAs. The identified rat miRNAs showed strong conservation with known human miRNAs in mirBase with only three unique miRNA seeds missing from known vertebrate homologs. Although these are unique and represent rat-specific sequences, 


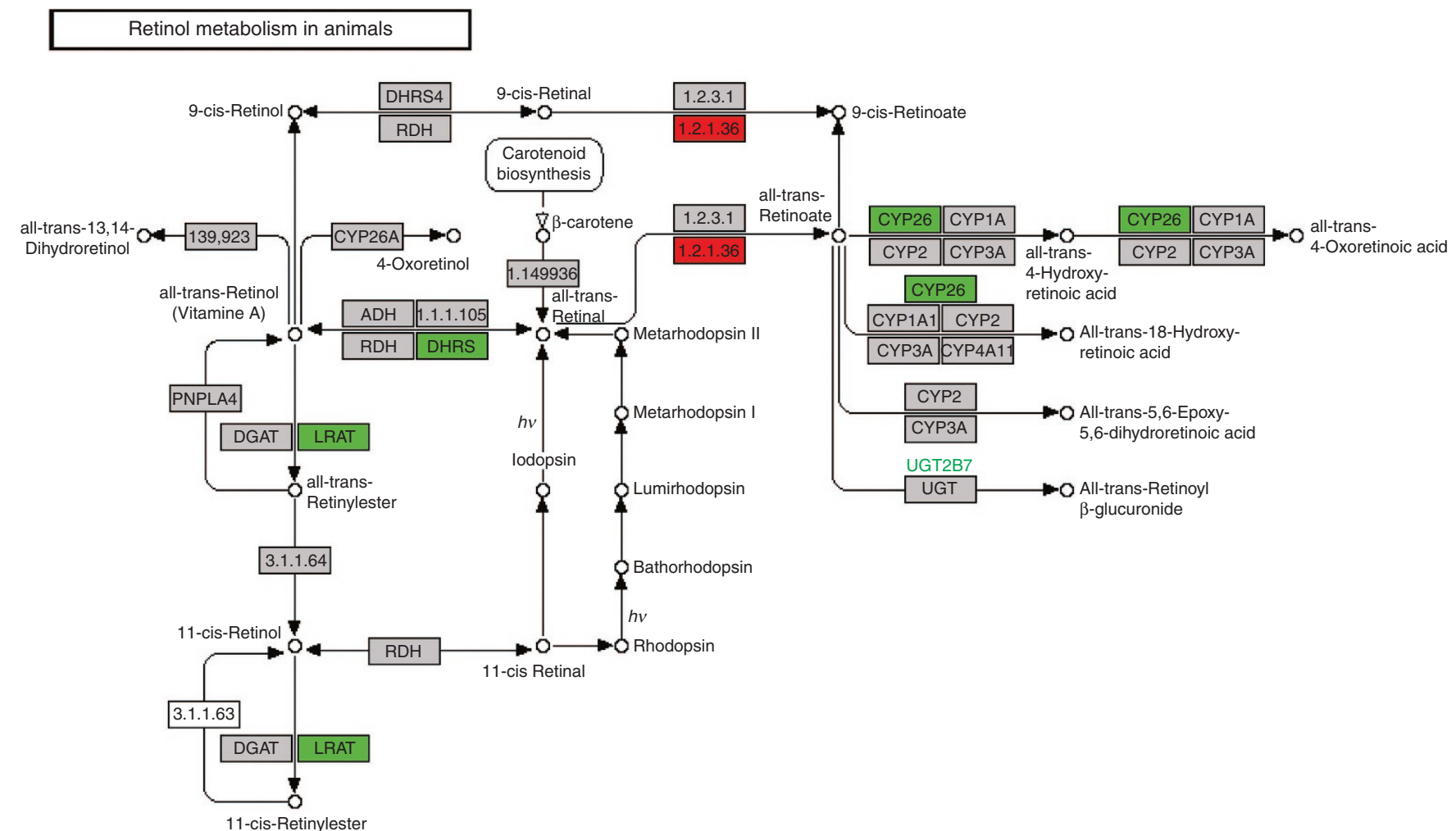

$0083011 / 12 / 13$

(c) Kanehisa Laboratories

Figure 2. Gene expression analysis comparing control and nitrofen-treated rat embryonic lungs as conducted through Kyoto Encyclopedia of Genes and Genomes (KEGG). Differentially expressed genes and enzymes are binomially labeled based upon their expression. Green indicates downregulated compared to control, while red indicates genes which are upregulated in the nitrofen-treated samples. Reprinted with permission from KEGG/Genome Net (http://www.kegg.jp/pathway/map00830).

we cannot exclude the possibility that they are unique in humans or remain to be detected in other species.

Interestingly, the density of miRNA genes is remarkably similar across the rat and human lineages. Putative miR-290, -500, -194 and -203 families in human tend to be organized in clusters, with large proportions having multiple isoforms and/or copies in their vicinity in the genome, suggesting that many of them could be a result of gene duplications or act redundantly. The rat gene encoding miR-291a: a gene linked to negative regulation of apoptotic processes and SMAD signaling has been previously identified as pluripotent embryonic stem cell-specific in mouse, rat, and in human, known as miR-hes-1, $-2,-3$ plays a role in embryonic stem cell differentiation (14). Our finding of a redundant expression of the six homologous hsa-290 isoforms that are orthologous to rat miRNA suggests that they are pluripotent embryonic stem miRNAs with crucial regulatory differentiation roles in early human development. One exception is the density of miRNA genes. The above results demonstrate that sequence conservation may point to conservation in function of human miRNAs that are not validated experimentally.

\section{Rat miRNAs and Their Target mRNAs Could Function Similarly to Their Human Homologs in Response to Nitrofen}

MiRNAs are derived from larger precursors that have the capacity to form stem-loop structures from either side of the strand.
Existing evidence based on the hairpin fold structure suggests phylogenetic conservation of our rno-miRNA data (15). For example, the orthologous hsa-miR-200c-3p that was enriched in the rat lung tissues and upregulated upon nitrofen treatment exhibited seed conservation with its host gene. Our findings, with regard to lung development, are consistent with increasing evidence from human studies on the effects of teratogenic and toxic triggers on miRNA profiling, and reconcile with orthologous studies matching the nitrofen-mediated upregulation of rat miR-101a-5p, -3p and human miR-144-5p in vivo in human airway epithelial cells upon cigarette smoke (16). Both of these miRNAs are also known to target genes associated with lung diseases such as the cystic fibrosis transmembrane conductor regulator gene in human (16). We also reported the upregulation of rat miR-193b after nitrofen exposure. Others attributed cystic fibrosis transmembrane conductor regulator replenishment and cancer regression to the upregulation of miR-193b in human (17). Thus, miR-193b may rescue cystic fibrosis transmembrane conductor regulator downstream of nitrofen in rat lung hypoplasia-a finding that warrants further investigation. We report a 4.04-fold increase in rno-miR-193-3p after nitrofen treatment and a high level of conservation with its human ortholog, demonstrating hsa-miR-193-3p to be a putative antiproliferative molecule that may play a role in $\mathrm{CDH}$ progression by a similar mechanism in human. 
Articles | Mahoodetal.

Table 1. Correlations of the microRNA (miRNA)-mRNA pairs

\begin{tabular}{|c|c|c|c|c|}
\hline $\begin{array}{l}\text { Pearson correlation } \\
\text { coefficient }\end{array}$ & $\begin{array}{l}\text { miRNA annotation } \\
\text { (mirBase) }\end{array}$ & $\begin{array}{l}\text { Fold change } \\
\text { (nitrofen/control) }\end{array}$ & $P$ value & Gene \\
\hline 1 & rno-miR-3585-5p & 2.90 & 0.01 & TPD52L1 \\
\hline 1 & rno-miR-142-5p & 0.63 & 0.00 & $\begin{array}{l}\text { ABHD2, BAZ2A, CYP26B1, EIF4E3, HIPK2, IKZF2, LRAT, } \\
\text { MAML1, ROBO1, SRGAP1 }\end{array}$ \\
\hline 1 & rno-miR-504 & 0.63 & 0.00 & RNF44 \\
\hline 1 & rno-miR-325-3p & 0.55 & 0.00 & $\begin{array}{l}\text { B3GNT7, CFTR, CNN1, FAM189B, FAM189B, FOXN3, GPR155, } \\
\text { IKZF2, INHA, LRRN1, MAML1, MUSTN1, NR5A2, TRAK1 }\end{array}$ \\
\hline 1 & rno-miR-615 & 0.43 & 0.00 & IKZF2 \\
\hline 1 & rno-miR-384-5p & 0.32 & 0.00 & $\begin{array}{l}\text { GCNT2, HIPK2, IKZF2, ITGB3, MAML1, MYH11, NR5A2, } \\
\text { PCDH20, R3HDM1, RNF44, SBK1, SCN8A }\end{array}$ \\
\hline 1 & rno-miR-216b-3p & 0.19 & 0.00 & FOXN3, IKZF2, SPRED2 \\
\hline 1 & rno-miR-291a-5p & 0.19 & 0.00 & BAZ2A, PARM1, PRKAA2, TGFBR3 \\
\hline-1 & rno-miR-592 & 4.50 & 0.00 & CYP26B1 \\
\hline-1 & rno-miR-877 & 3.99 & 0.00 & FOXN3 \\
\hline-1 & rno-miR-215 & 3.43 & 0.00 & ABHD2 \\
\hline-1 & rno-miR-3585-5p & 2.90 & 0.01 & BAZ2A, IKZF2, ITGB3, SIRPA, SPRY4, SRGAP1, TGFBR3 \\
\hline-1 & rno-miR-484 & 2.38 & 0.00 & FOXN3, PCDHGA8, TGFBR3 \\
\hline-1 & rno-miR-369-3p & 2.12 & 0.00 & ADCY5, AHDC1, CYP26B1, FOXN3, GREM2, IKZF2 \\
\hline-1 & rno-miR-142-5p & 0.63 & 0.00 & DNAL1 \\
\hline-1 & rno-miR-504 & 0.63 & 0.00 & PDE1B \\
\hline-1 & rno-miR-325-3p & 0.55 & 0.00 & $\begin{array}{l}\text { FIBIN, GADD45G, MASP1, MRPS25, MYO7B, PDE1B, PPCS, } \\
\text { TMEM205 }\end{array}$ \\
\hline-1 & rno-miR-384-5p & 0.32 & 0.00 & ADO, ATP2B2, DCUN1D2 \\
\hline-1 & rno-miR-216b-3p & 0.19 & 0.00 & ID4 \\
\hline
\end{tabular}

Genes expressed in the lung are in bold. The $P$ value indicates the likelihood of the focus genes in a network being found together by random chance. The same computation was used for GO analysis of the initial gene list. The columns are sorted in descending order of $P$ value.

Please refer to Supplementary Table $\$ 2$ online for full names of the gene symbols.

We report a $>$ twofold increase in the expression of another highly conserved miRNA; let-7d-3p in nitrofen-induced hypoplastic lungs. This result is in contrast to Pandit and colleagues (18), who demonstrated that a lack of let-7d promotes alveolar thickening of alveolar compartments exclusively. The latter study demonstrated that a lack of let-7d causes epithelial-to-mesenchymal transition in lung epithelial cells from idiopathic pulmonary fibrosis patients, mediated by TGF- $\beta$ by a SMAD-dependent mechanism. Nitrofen induced rno-let-7b-5p by 3.89 -fold and $-7 \mathrm{~d}-3 p$ by 2.27 -fold, that target the Ras transcripts downstream of the fibroblast growth factor receptors (19), thus downregulating lung branching and epithelial cell proliferation. Mir-145 consists of a specific miRNA signature associated with pulmonary hypertension and regulates airway smooth muscle cell differentiation according to previous studies (20). Our results indicate that miRNA rno-miR-145-3p and $-5 p$ is a putative component in $\mathrm{CDH}$ abnormal lung development in both rat and humans, corroborating the results from others (20). Consequently, future studies should target miR-145 to investigate if it plays a role in persistent pulmonary hypertension in $\mathrm{CDH}$.

\section{miRNAs Mediate Nitrofen-induced Cell Cycle Arrest and Proliferation Inhibition}

Most differentially expressed genes from the nitrofen lungs are clustered, demonstrating a strong expression profile in the developing lung. Nitrofen persists in the lung at elevated levels for 3 days and hence a single dose is enough for inducing intense irreversible damage to the cells (5). Nitrofen induces genotoxicity that modulates several miRNAs, and thereby alters critical cell cycle control signaling networks. We and others have previously reported in both the nitrofen rat and mouse model that cell cycle-associated proteins are downregulated in nitrofen-induced lung hypoplasia $(8,21,22)$. Nitrofen has been demonstrated to suppress cell proliferation by inhibiting regulators of DNA replication (8), activation of the mitochondrial apoptosis axis $\mathrm{Bcl} 2$, Bax, and Bak, inhibition of $\mathrm{Bcl}-\mathrm{xl}$, and executing the activation of $\mathrm{p} 38$-mitogen-activated protein kinase P-38 MAPK in cultured type II pneumocytes (23). ABHD2 plays an important role in maintaining lung structure integrity and is expressed in alveolar type II cells in mice lungs. In mouse pulmonary smooth muscle cells, ABHD2 triggers the differentiation of monocytes to macrophages. Its deficiency often presents emphysema-like symptoms including increased macrophage infiltration, increased inflammatory cytokines, a protease/anti-protease imbalance and enhanced apoptosis in a strikingly similar fashion to human pathology (24). Here, we identified a nitrofen-perturbed immune response pattern in the lung and implicated a role for ABHD2. Similarly to nitrofen, the lung carcinogen benzo(a)pyrene downregulates miR$142-5$ p significantly (25). 


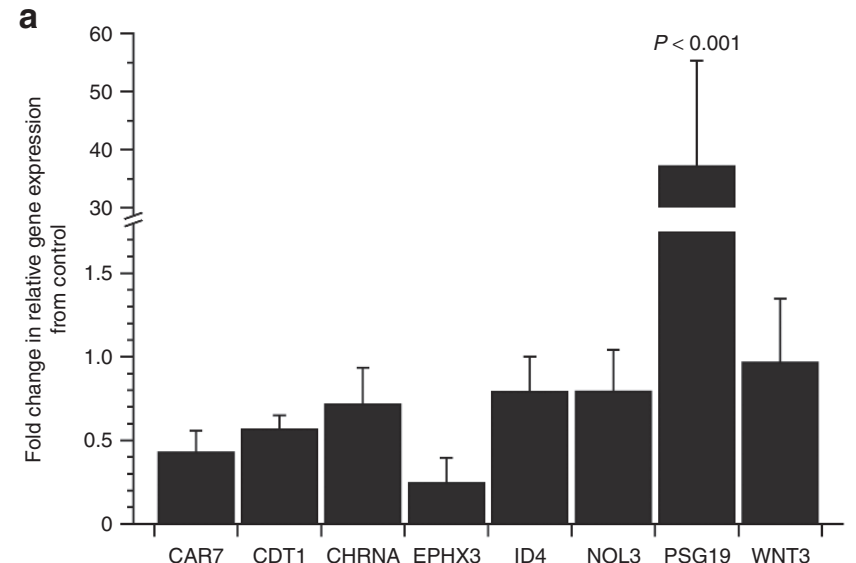

C

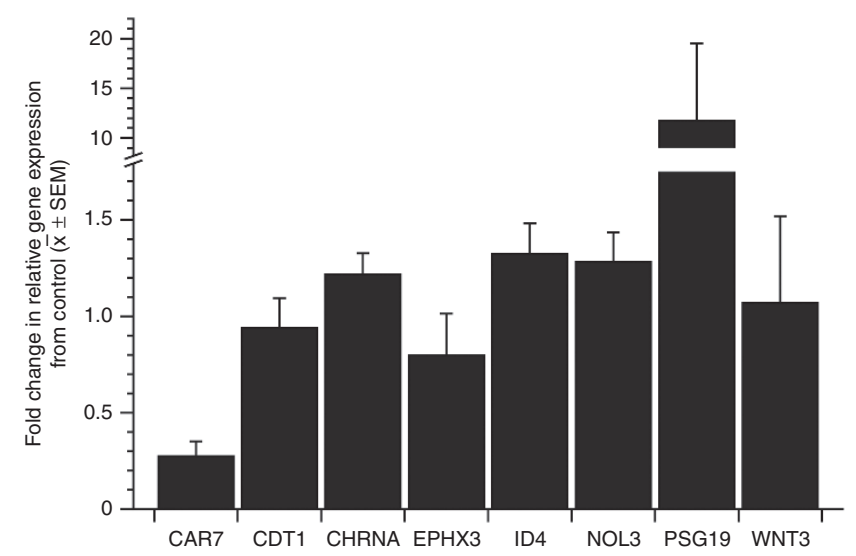

b

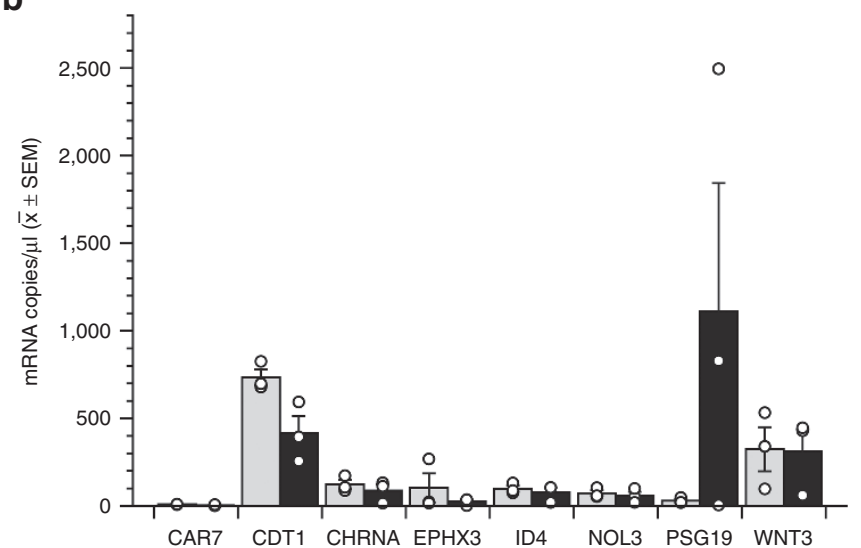

d

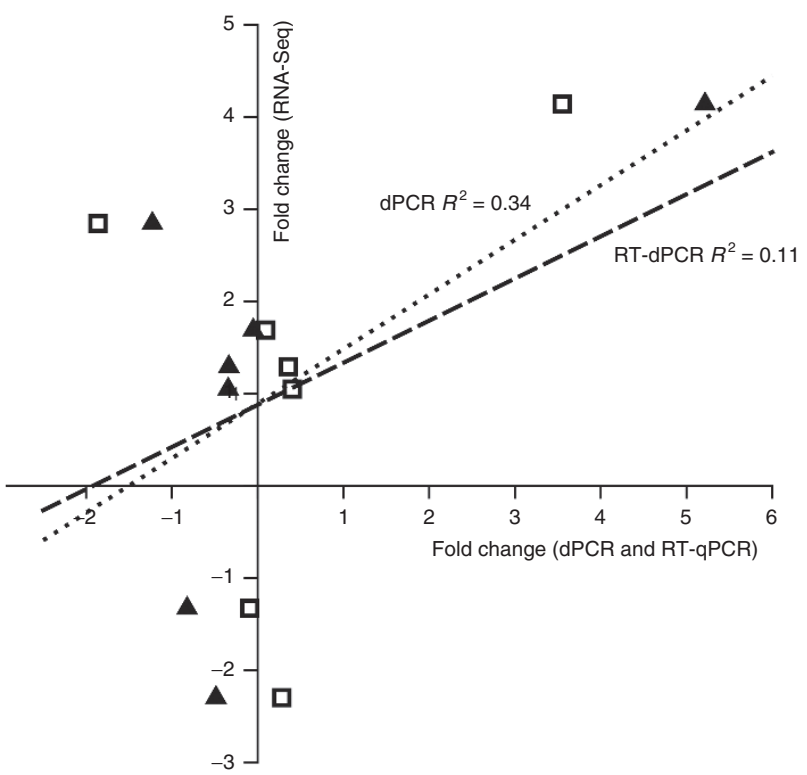

Figure 3. Next-generation sequencing (NGS) validation by digital PCR and RT-qPCR. Validation of the expression of a subsample of genes by dPCR. Two-way ANOVA $(P<0.001)(\mathbf{a}, \mathbf{b})$. RT-qPCR of the selected NGS transcripts with no significant changes were shown (PSG19 two-tailed $t$-test $(P=0.0558)$ (c). (d) Linear regression correlation of RNA-seq ( $y$ axis) with dPCR and RT-qPCR data ( $x$ axis) using a log 2 mean-fold change measure of the genes differentially expressed across the two gene-expression platforms under correlation analysis. Outliers (Ephx3) were identified and removed as having 1.5 SD or larger. Grey indicates control, black indicates nitrofen-treated samples, circles indicates biological replicates, triangles represent dPCR results, and open squares indicate RT-qPCR results.

In the current study, rno-miR-325-3p positively correlated with nitrofen-induced GPR155 and NR5A2. GPR155 is an integral membrane protein related to G-protein-coupled receptors. The mRNAs for GPR155 are widely expressed in adult mouse tissues and during the development of the lung and brain. RnomiR-384-5p and NR5A2 expressions correlate positively upon nitrofen exposure. MiR-384-5p is a marker of neurotoxicity and regulates apoptotic cell death in myocardial ischemia by modulating PIK3CD pathway (26). Myosin heavy chain 11 is a component of the contractile apparatus of aortic smooth muscle cells and is expressed in neonatal lung smooth muscle cells (27). RNF44 is a poorly understood protein that is encoded by a gene and contains a RING finger motif, a motif often present on proteins involved in protein-protein and protein-DNA interactions. In the current study, nitrofen induced both myosin heavy chain 11 and RNF44 in embryonic lungs, and their expression positively correlated with miR-384-5P expression. These data suggest a potential role for this pathway in the vascular remodeling observed in $\mathrm{CDH}$-associated pulmonary hypertension.

\section{miRNAs Regulate Nitrofen-induced Airway Smooth Muscle Cell Dysfunction}

Airway smooth muscle cells (ASMC) progenitors in the embryonic lung mesenchyme produce fibroblast growth factor-10 required for epithelial cell morphogenesis. Fibroblast growth factor-10 expression is crucial for fine tuning the spatiotemporal ASMC activity early in human development. The relationship between lung hypoplasia, fibroblast growth factor-10 deficiency, and dysfunctional ASMC from birth onward is linked (28), and as a perturbation of this relationship by nitrofen results in abnormal smooth muscle relaxation near term. Calponin (CNN1), a multifunctional 


\section{Articles | Mahoodet al.}

orthologous protein in both rats and humans in ASMC, is tightly restricted to differentiated ASMC lineages including those in the lung during embryonic and postnatal life. In the current study, nitrofen downregulates CNN1, the latter correlates with the miR-504 upregulated expression.

E-cadherin and integrin are major components of the extracellular matrix. Loss of E-cadherin is a hallmark of epithelial-to-mesenchymal transition and synergizes the dedifferentiation capacity of the cell. From our study, we identified a nitrofen-induced upregulation of rno-miR-296-3p. MiR$192 / 215$ has been shown to mediate changes in E-cadherin expression via targeting the ZEB2 family of transcriptional repressors downstream of TGF- $\beta$ (29). In addition, our study showed downregulation of members of the miR-200 family (rno-miR-200c-3p, rno-miR-200c-5p, and rno-miR-200b-5p) upon nitrofen treatment by $>$ twofold relative to the control. Expression of miR-200 and ZEB1/2 is linked to a negative feedback loop (30) that may involve rno-miR-200c-3p, -200b-5p in a crosstalk with TGF- $\beta$. A previous study from Xu et al. (31) using immunohistochemistry in the nitrofen rat model demonstrated that TGF- $\beta 1$ protein was stronger in $\mathrm{CDH}$ lungs.

Retinoic acid (RA) signaling is essential for fetal lung development and postnatal alveolar septation (32). Nitrofen suppresses the retinoid response element by a mechanism yet to be identified (33) and this suppression is reversible by RA supplementation. CYP26B1 gene encodes cytochrome P450, an RA metabolizing enzyme that is expressed in embryonic lung tissues undergoing morphogenesis. CYP26B1 fine tunes the activity of RA. The primary function of lecithin retinol acyltransferase is to convert retinol to the more functional retinyl esters. We report that nitrofen inhibits CYP26B1 and lecithin retinol acyltransferase. Studies have shown that inhibiting CYP26 augments RA function in human epidermal keratinocytes (34). The above data corroborates with previous findings that RA is diminished upon nitrofen exposure. We find that CYP26B1 and lecithin retinol acyltransferase correlate positively with $\mathrm{miR}-142-5 \mathrm{p}$ expression, whereas miR592 negatively correlates with CYP26B1 expression. These results confirm the previous evidence from clinical studies that showed retinol levels to be lower in babies born with $\mathrm{CDH}$ (35). MiR-10a is an RA target (36) and is localized in the vicinity of the HOXB cluster of chromosome 17 in humans. The HOXB locus is involved in lung branching morphogenesis and has a putative role in determining cell fate decision in the lung (37). Our study identified nitrofen-mediated upregulation of rno-miR-10b. MiR-10a (a close homolog of MiR$10 \mathrm{~b}$ ) is a known mediator of RHOC, and functions through the upstream axis TWIST-miR-10b-HOXD10-RHOC (38). $\mathrm{PI} 3 \mathrm{~K} / \mathrm{mTOR}$ signaling is required for miR-10 activity and depends on their interactions with $5^{\prime}$ TOP mRNAs and cell type (39). Doi et al. (40) previously reported that PI3K mRNA is decreased in E21 nitrofen-induced hypoplastic lungs. They also showed with immunohistochemistry that PI3K protein was diminished in the distal epithelium of E18 CDH lungs and that PI3K overall intensity was decreased in E21 CDH lungs (40).
Our NGS analysis demonstrated that rno-miR-142-5p and -291a-5p are differentially expressed and correlate positively with BAZ2A, a chromatin-dependent transcriptional regulator-an integral component of chromatin re-modeling complexes. We report that rno-miR-3585-5p negatively alters two genes; BAZ2A and SIRPA; however, the exact mechanism is poorly understood. It has been shown that SIRPA, a member of the signalregulatory-protein family, can interact with surfactant proteins $\mathrm{A}$ and $\mathrm{D}$ to suppress alveolar phagocytosis without triggering an inflammatory response (41). This protein can be phosphorylated by tyrosine kinases and is found to participate in signal transduction triggered by CD47 and mediated by various growth factor receptors.

\section{Limitations and Future Research}

One of the limitations of our study is the use of the teratogen nitrofen to induce lung hypoplasia and $\mathrm{CDH}$. Even though this model has been widely used and accepted to study the pathogenesis of abnormal lung development and CDH (reviewed by us in (5)), a direct link between nitrofen use and abnormal lung development and $\mathrm{CDH}$ in humans has never been established.

When performing a transcriptomic analysis, there are limitations that are inherent to the design of such a study. We have shown that several genes and miRNAs are involved in nitrofeninduced abnormal lung development and CDH. How these transcript differences translate to changes in protein structure and function remains unknown. A high-throughput proteomic survey combined with immunoblotting candidate proteins would be ideal to assess these differences. It is possible that the sampling differences between the NGS study and that conducted by the biological replicates could be the strongest effect being shown. The RNA used for the experiment was pooled from multiple dams whereas RNA used for the functional gene expression analysis was true biological replicates derived from multiple dams. These biological replicates are essential as nitrofen induces a $\mathrm{CDH}$-like lung phenotype in up to $100 \%$ of E13.5 rats. Due to this uncertainty in the developmental phenotype, the variability that would be induced at the genetic level due to nitrofen would be more pronounced than shown in the phenotypic presentation of nitrofen-induced $\mathrm{CDH}$. This biological bias combined with the technical variability in NGS complicates the functional assessment of the NGS results. Further extrapolations into the transcriptomic effects of nitrofen warrant a stronger presence of biological diversity amongst samples. An important followup study would be a combined miRNA and mRNA sequencing experiment across consecutive stages of lung development. In our study, the age-matched embryonic tissues and the nature of the collected samples limited our primary material and thus we had to pool multiple lungs for analysis. This approach did not permit the proper assessment of biological variation. Additionally, using a mixed sample approach studying a highly heterogeneous organ such as the lung does not permit focusing on functionally distinct regions or substructures, making the establishment of expression profiles less informative. We could not confirm tissue-specific, strain-specific or cell-specific differential miRNA or gene expression pattern for all reads analyzed. This would have 


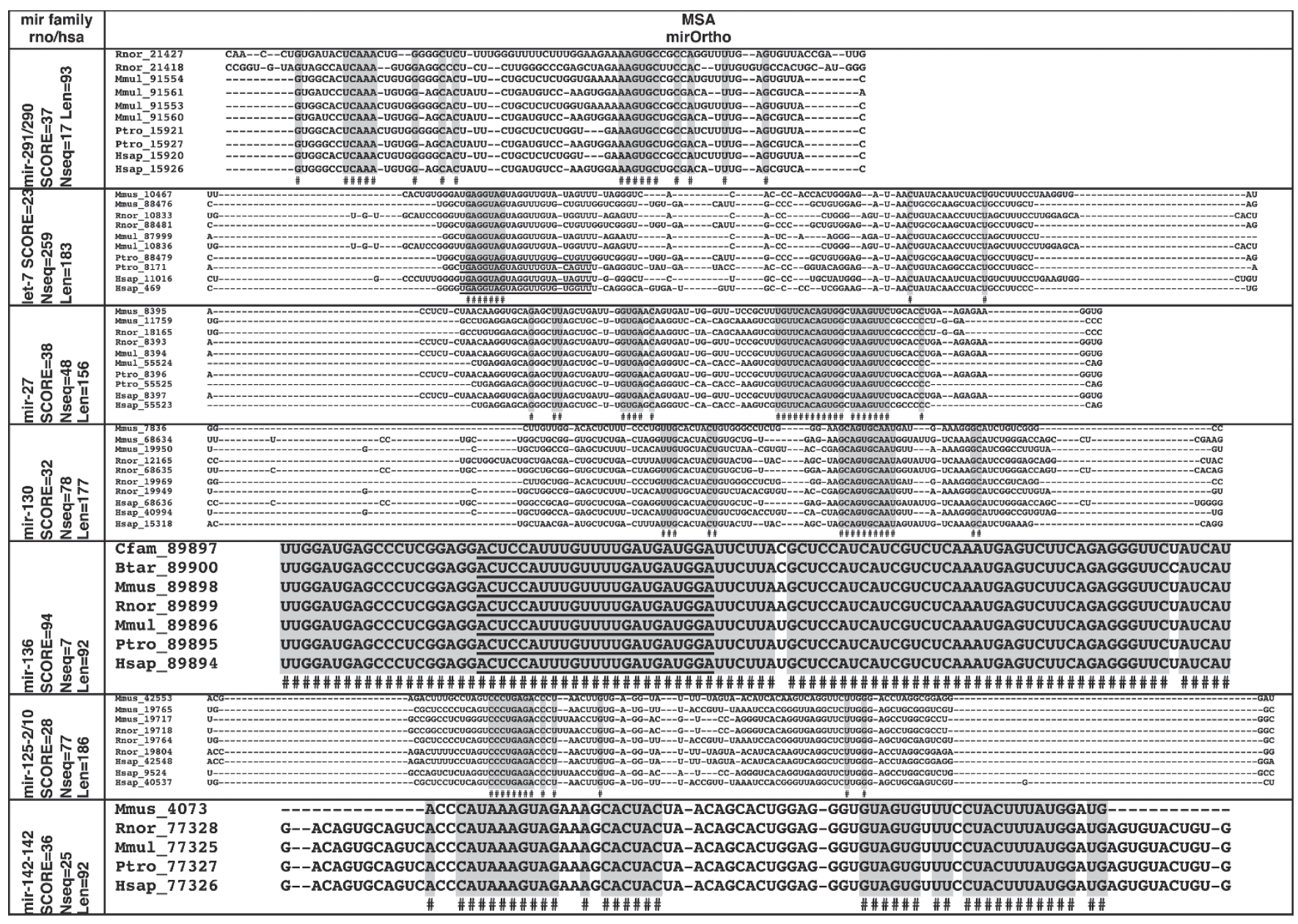

Figure 4. Multiple sequence alignments and structure conservation of functionally relevant embryonic and fetal miRNA orthologuos sequences cross species. CLUSTAL format for T-COFFEE version 6.92 using the default parameters (46). Sequence conservation is represented by asterisks and shaded, mature miRNAs are underlined. Species ID are provided by the mirOrtho database.

been an important step in elucidating miRNA functions. Future studies analyzing cell type differences across the various tissues and linking cell types to transcriptome changes would provide a better understanding at which level these changes have an effect.

Although our study does not address whether altered mRNA expression is a cause or an effect of miRNA-altered expression, the presence of a relationship between the two provides a starting point for future studies. Further experimental work including luciferase reporter gene assays and mutagenesis of predicted miRNA-binding sites will be useful to examine these interactions further.

\section{Closing Remarks}

Our study characterizes the transcriptome associated with nitrofen-induced abnormal lung development using NGS technology. We identified several miRNAs, including members of the miR-290, $-500,-194,-203,-200 c,-200 b,-101 \mathrm{a}$, $-144,-193,-145,-142,-215,-384,-504,-10 \mathrm{a}$ and -296 families, that deserve further investigation. Our data highlight the importance of PI3K, TGF- $\beta$, RA, and cell cycle kinase signaling in nitrofen-induced rat lung hypoplasia.

\section{METHODS}

Animals, Nitrofen Administration and Sample Preparation

The University of Manitoba Animal Care Committee approved all animal care protocols. The nitrofen model was used as described before. A total of two pregnant dams were included in each group. Embryonic (E)13.5 lungs were isolated and snap frozen in liquid nitrogen. Total RNA was extracted from pools of four lungs for each sample using the mirVana extraction kit (ThermoFisher, Waitman, MA). DNA contamination was removed with a DNase treatment kit (Ambion Life Technologies, Carlsbad, CA) before yield was determined spectrophotometrically. RNA quality was assessed using a Bioanalyzer (Agilent Technologies, Santa Clara, CA) with RNA integrity values of greater than 9 used for further analysis (see Supplementary Figure S3 online).

Small RNAseq (miRNA) Library Preparation and Sequencing

Total RNA $(1 \mu \mathrm{g})$ was quantitated by the Qubit fluorometer (ThermoFisher) and the quality was evaluated using a Bioanalyzer (Agilent Technologies). After $5^{\prime}$ and $3^{\prime}$ adapter ligation, transcripts were amplified with barcoded primers for parallel sample sequencing. Samples were combined and run on a polyacrylamide gel and miRNAs and piwi interacting RNAs were cut out of the gel and purified using standard molecular biology techniques. The miSeq library was then validated by RT-qPCR before running on an Illumina MiSeq next-generation sequencing instrument (Illumina, San Diego, CA) using 50 base pairs single end reads. 


\section{Total RNAseq Library Preparation and Sequencing}

Using $5 \mu \mathrm{g}$ of ribosomal depleted total RNA (Epicenter Ribo-Zero, Illumina), the total RNA library was created using the SOLiD total RNA-Seq kit (ThermoFisher). Briefly, the RNA was fragmented to 100-200 bp using RNAse III before being hybridized and ligated to SOLiD adapters. The library was reverse transcribed to cDNA before being size selected using AmPure XP beads (Beckman Coulter, Mississauga, Canada). Once cDNA libraries were barcoded (SOLiD primers, ThermoFisher) and assessed (Agilent Technologies), libraries were pooled and run for next-generation sequencing on the SOLiD 5500xl instrument (ThermoFisher).

\section{Bioinformatics and Statistical Analysis}

SOLiD RNAseq data were mapped to the $R$. norvegicus reference genome (rn5) using Lifescope v2.5.1 software (ThermoFisher) with the two-mismatch settings. The mapped reads were quantified against the University of Santa Cruz-annotated refGenes and normalized gene expression profiles, reads per kilobase per million reads, were generated. The bioconductor package edgeR (42) based on a negative binomial model was used to infer the differentially expressed genes between the treated and control samples. MiSeq sequence reads were used for miRNA profiling. After adaptor removal and quality check, the differentially expressed mature miRNAs were analyzed using the mirTools 2.0 pipeline (43). The rno mirBase v16 with "best match" setting was used. We detected 100 miRNAs by a cutoff of $P \leq 0.01$ and fold change (FC) $\geq 1.5$ and their mature sequences were further identified using mirBase. The miRNA and mRNA expression data files of the nitrofentreated and control lung tissue samples were loaded into the Partek Genomics Suite v6.6 (Partek Inc, St Louis, MO). The Pearson correlations of the mirRNAs and their target mRNAs were analyzed against the rno-miR database using the miRNA integration module. For differentially expressed miRNAs, all sequence reads in rat and mRNA targets overlapping with differentially expressed genes were used to perform IPA pathway analysis to obtain a list of enriched pathways (Ingenuity Systems, Redwood City, CA). We identified human ortholog sequences by mapping the short (rno) reads to a known miRNA database (mirBase) using BLASTN and other tools from the mirOrtho database (44) (see Supplementary Table S3 online). We identified homolog sequences of selected miRNAs among different species using the stem loop structure. Consequently, the aligned sequence conservation of miRNAs was compared across species (see Supplementary Table S4 online and Figure 4). Differential expression of miRNA between paired samples was measured using a Chi-squared statistic. $P$ values were calculated for the null hypothesis that no differential expression existed between the two samples. Final $P$ values are corrected using the Bonferroni correction for multiple hypothesis testing.

\section{Endogenous Control TaqMan Assay}

Embryonic rat lungs (E21) were isolated from three different dams, and total RNA was extracted and reverse transcribed as described previously. A rat endogenous control plate (ThermoFisher) was seeded with cDNA ( $25 \mathrm{ng}$ RNA input per reaction), TaqMan Fast Advanced Master Mix (ThermoFisher), and water to $10 \mu \mathrm{L}$. The plates were run on a CFX96 thermocycler (Bio-Rad, Mississauga, Canada) using the recommended cycling parameters and Raw $C_{\mathrm{t}}$ values were reported along with $\mathrm{m}$-value analysis provided by the accompanied software (CFX Manager 3.1; Bio-Rad).

\section{Primer Design and Optimization}

Briefly, primers were designed using Primer Quest (Integrated DNA Technologies, Coralville, IA) and verified for specificity using UNAFold (IDT), and Primer-Blast (45) software (see Supplementary Table S5 online). Primer specificity was verified using conventional molecular techniques before being optimized for SYBR Green annealing temperature and melting curve specificity using gradient RT-qPCR and also determined with digital-PCR (dPCR).

\section{Digital PCR}

Samples were diluted to an optimal concentration $(1.6$ copies $/ \mu \mathrm{L}$ in water) using an Early Access Digital PCR Experiment Design application (ThermoFisher). Each sample was combined with QuantStudio 3D Master Mix (ThermoFisher), SYBR I (ThermoFisher), primers $(500 \mathrm{nM}$ each) and built up to $14.5 \mu \mathrm{L}$ with water. Reactions were loaded, filled, and sealed on separate QuantStudio 3D Digital PCR 20k Chips (ThermoFisher) and run on a QuantStudio 3D chip reader (ThermoFisher) with data analysis completed using the associated online software.

\section{RT-qPCR}

Using SsoAdvanced SYBR green supermix (Bio-Rad), each reaction well was loaded with primers (500 nM each), along with $10 \mathrm{ng}$ of template and built up to $20 \mu \mathrm{L}$ with water. Samples run using a CFX 96 thermocycler with the following cycling parameters: $95^{\circ} \mathrm{C} 30 \mathrm{~s}, 40$ cycles of $95^{\circ} \mathrm{C}$ for $15 \mathrm{~s}$ and annealing at $59^{\circ} \mathrm{C}$ for $30 \mathrm{~s}$ after which a melting curve was performed. Two endogenous controls as determined by the TaqMan assay were used to calculate changes in expression (CFX Manager 3.1, Bio-Rad).

\section{SUPPLEMENTARY MATERIAL}

Supplementary material is linked to the online version of the paper at http:// www.nature.com/pr

\section{ACKNOWLEDGMENTS}

We would like to thank Dr. Deborah Tsuyuki, Manager of the Next Generation Sequencing Platform at the Children's Hospital Research Institute of Manitoba for her technical assistance. This work was supported by a small grant from the Children's Hospital Research Institute of Manitoba. Richard Keijzer holds a career enhancement award from the Canadian Child Health Clinician Scientist Program and a New Investigator Salary Award from the Canadian Institutes of Health Research and Manitoba Lung Association/Children's Hospital Research Institute of Manitoba.

Disclosure: The authors have no conflict of interest relevant to the subject matter of this manuscript.

\section{REFERENCES}

1. Inui M, Martello G, Piccolo S. MicroRNA control of signal transduction. Nat Rev Mol Cell Biol 2010;11:252-63.

2. Yates LA, Norbury CJ, Gilbert RJ. The long and short of microRNA. Cell 2013;153:516-9.

3. Johar D, Siragam V, Mahood TH, Keijzer R. New insights into lung development and diseases : the role of microRNAs. 2015;10:1-10.

4. Keijzer R, Puri P. Congenital diaphragmatic hernia. Semin Pediatr Surg 2010;19:180-5

5. van Loenhout RB, Tibboel D, Post M, Keijzer R. Congenital diaphragmatic hernia: comparison of animal models and relevance to the human situation. Neonatology 2009;96:137-49.

6. Manson JM. Mechanism of nitrofen teratogenesis. Environ Health Perspect 1986;70:137-47.

7. Allan DW, Greer JJ. Pathogenesis of nitrofen-induced congenital diaphragmatic hernia in fetal rats. J Appl Physiol (1985) 1997;83:338-47.

8. Keijzer R, Liu J, Deimling J, Tibboel D, Post M. Dual-hit hypothesis explains pulmonary hypoplasia in the nitrofen model of congenital diaphragmatic hernia. Am J Pathol 2000;156:1299-306.

9. Kent WJ, Sugnet CW, Furey TS, et al. The human genome browser at UCSC. Genome Res 2002;12:996-1006.

10. Mi H, Muruganujan A, Thomas PD. PANTHER in 2013: modeling the evolution of gene function, and other gene attributes, in the context of phylogenetic trees. Nucleic Acids Res 2013;41 (Database issue):D377-86.

11. Kanehisa M, Goto S, Sato Y, Kawashima M, Furumichi M, Tanabe M. Data, information, knowledge and principle: back to metabolism in KEGG. Nucleic Acids Res 2014;42(Database issue):D199-205.

12. Kozomara A, Griffiths-Jones S. MiRBase: annotating high confidence microRNAs using deep sequencing data. Nucleic Acids Res 2014;42:88-91.

13. Bhaskaran M, Wang $\mathrm{Y}$, Zhang $\mathrm{H}$, et al. MicroRNA-127 modulates fetal lung development. Physiol Genomics 2009;37:268-78.

14. Houbaviy HB, Murray MF, Sharp PA. Embryonic stem cell-specific microRNAs. Dev Cell 2003;5:351-8.

15. Ambros V, Bartel B, Bartel DP, et al. A uniform system for microRNA annotation. RNA 2003;9:277-9.

16. Hassan F, Nuovo GJ, Crawford M, et al. MiR-101 and miR-144 regulate the expression of the CFTR chloride channel in the lung. PLoS One 2012;7 (11): e50837. 
17. Xie C, Jiang XH, Zhang JT, et al. CFTR suppresses tumor progression through miR-193b targeting urokinase plasminogen activator (uPA) in prostate cancer. Oncogene 2013;32:2282-91, 2291.e1-7.

18. Pandit KV, Corcoran D, Yousef $\mathrm{H}$, et al. Inhibition and role of let-7d in idiopathic pulmonary fibrosis. Am J Respir Crit Care Med 2010;182:220-9.

19. Johnson SM, Grosshans H, Shingara J, et al. RAS is regulated by the let-7 microRNA family. Cell 2005;120:635-47.

20. Davis-Dusenbery BN, Chan MC, Reno KE, et al. Down-regulation of Kruppel-like factor-4 (KLF4) by microRNA-143/145 is critical for modulation of vascular smooth muscle cell phenotype by transforming growth factorbeta and bone morphogenetic protein 4. J Biol Chem 2011;286:28097-110.

21. Guilbert TW, Gebb SA, Shannon JM. Lung hypoplasia in the nitrofen model of congenital diaphragmatic hernia occurs early in development. Am J Physiol Lung Cell Mol Physiol 2000;279:L1159-71.

22. Chinoy MR, Chi X, Cilley RE. Down-regulation of regulatory proteins for differentiation and proliferation in murine fetal hypoplastic lungs: altered mesenchymal-epithelial interactions. Pediatr Pulmonol 2001;32:129-41.

23. Tong QS, Zheng LD, Tang ST, et al. Nitrofen suppresses cell proliferation and promotes mitochondria-mediated apoptosis in type II pneumocytes. Acta Pharmacol Sin 2007;28:672-84.

24. Jin FG, Li WP, Mu DG, et al. Joint application of 7 interventional pulmonology methods in early diagnosis of lung cancer. Zhonghua Yi Xue Za Zhi 2009;89:1667-71.

25. Halappanavar S, Wu D, Williams A, et al. Pulmonary gene and microRNA expression changes in mice exposed to benzo(a)pyrene by oral gavage. Toxicology 2011;285:133-41.

26. Bao W, Holt LJ, Prince RD, et al. Novel fusion of GLP-1 with a domain antibody to serum albumin prolongs protection against myocardial ischemia/ reperfusion injury in the rat. Cardiovasc Diabetol 2013;12:148.

27. Popova AP, Bozyk PD, Goldsmith AM, et al. Autocrine production of TGFbeta1 promotes myofibroblastic differentiation of neonatal lung mesenchymal stem cells. Am J Physiol Lung Cell Mol Physiol 2010;298:L735-43.

28. Jesudason EC, Smith NP, Connell MG, et al. Peristalsis of airway smooth muscle is developmentally regulated and uncoupled from hypoplastic lung growth. Am J Physiol Lung Cell Mol Physiol 2006;291:L559-65.

29. Wang B, Herman-Edelstein $M$, Koh $P$, et al. E-cadherin expression is regulated by miR-192/215 by a mechanism that is independent of the profibrotic effects of transforming growth factor-beta. Diabetes 2010;59: 1794-802.

30. Gregory PA, Bert AG, Paterson EL, et al. The miR-200 family and miR-205 regulate epithelial to mesenchymal transition by targeting ZEB1 and SIP1. Nat Cell Biol 2008;10:593-601.

31. Xu C, Chen ZX, Liu WY, Wang YX, Xiong ZX. A series of observation on the expression of TGF-betal in the lung of nitrofen-induced congenital diaphragmatic hernia rat model. Zhonghua Wai Ke Za Zhi 2009;47:301-4.
32. Massaro D, Massaro GD. Invited Review: pulmonary alveoli: formation, the "call for oxygen," and other regulators. Am J Physiol Lung Cell Mol Physiol 2002;282:L345-58.

33. Chen MH, MacGowan A, Ward S, Bavik C, Greer JJ. The activation of the retinoic acid response element is inhibited in an animal model of congenital diaphragmatic hernia. Biol Neonate 2003;83:157-61.

34. Pavez Loriè E, Li H, Vahlquist A, Törmä H. The involvement of cytochrome p450 (CYP) 26 in the retinoic acid metabolism of human epidermal keratinocytes. Biochim Biophys Acta 2009;1791:220-8.

35. Beurskens LW, Tibboel D, Lindemans J, et al. Retinol status of newborn infants is associated with congenital diaphragmatic hernia. Pediatrics 2010;126:712-20.

36. Weiss FU, Marques IJ, Woltering JM, et al. Retinoic acid receptor antagonists inhibit miR-10a expression and block metastatic behavior of pancreatic cancer. Gastroenterology 2009;137:2136-45.e1-7.

37. Volpe MV, Martin A, Vosatka RJ, Mazzoni CL, Nielsen HC. Hoxb-5 expression in the developing mouse lung suggests a role in branching morphogenesis and epithelial cell fate. Histochem Cell Biol 1997;108:495-504.

38. Ma L, Teruya-Feldstein J, Weinberg RA. Tumour invasion and metastasis initiated by microRNA-10b in breast cancer. Nature 2007;449:682-8.

39. Tang H, Hornstein E, Stolovich $\mathrm{M}$, et al. Amino acid-induced translation of TOP mRNAs is fully dependent on phosphatidylinositol 3-kinase-mediated signaling, is partially inhibited by rapamycin, and is independent of S6K1 and rpS6 phosphorylation. Mol Cell Biol 2001;21:8671-83.

40. Doi T, Ruttenstock E, Dingemann J, Puri P. Spatiotemporal alteration in phosphatidylinositide 3-kinase-serine/threonine protein kinase B signaling in the nitrofen-induced hypoplastic lung. J Pediatr Surg 2010;45: 366-71.

41. Janssen WJ, McPhillips KA, Dickinson MG, et al. Surfactant proteins A and D suppress alveolar macrophage phagocytosis via interaction with SIRP alpha. Am J Respir Crit Care Med 2008;178:158-67.

42. Robinson MD, McCarthy DJ, Smyth GK. edgeR: a bioconductor package for differential expression analysis of digital gene expression data. Bioinformatics 2010;26:139-40.

43. Zhu E, Zhao F, Xu G, et al. mirTools: microRNA profiling and discovery based on high-throughput sequencing. Nucleic Acids Res 2010;38(Web Server issue):W392-7.

44. Gerlach D, Kriventseva EV, Rahman N, Vejnar CE, Zdobnov EM. miROrtho: computational survey of microRNA genes. Nucleic Acids Res 2009;37(Database issue):D111-7.

45. Ye J, Coulouris G, Zaretskaya I, Cutcutache I, Rozen S, Madden TL. Primer-BLAST: a tool to design target-specific primers for polymerase chain reaction. BMC Bioinformatics 2012;13:134.

46. Notredame C, Higgins DG, Heringa J. T-Coffee: a novel method for fast and accurate multiple sequence alignment. J Mol Biol 2000;302:205-17. 